\title{
Bilateral Orbital Cellulitis with Pan Sinusitis in a Female without Cavernous Sinus Thrombosis- A Rare Presentation
}

\author{
Atma Nayak ${ }^{1}$, Sourya Acharya ${ }^{2}$, Aishwarya Ghule $^{3}$
}

\begin{abstract}
1Department of Medicine, Datta Meghe Institute of Medical Sciences (Deemed to be University) Sawangi, Meghe, Wardha, Maharashtra, India. ${ }^{2}$ Department of Medicine, Datta Meghe Institute of Medical Sciences (Deemed to be University) Sawangi, Meghe, Wardha, Maharashtra, India. ${ }^{3}$ Department of Medicine, Datta Meghe Institute of Medical Sciences (Deemed to be University) Sawangi, Meghe, Wardha, Maharashtra, India.
\end{abstract}

\section{INTRODUCTION}

Inflammation of the ocular structures behind the orbital septum is called orbital cellulitis. Etiological agents are usually bacteria and fungi. It affects persons of all ages but has greater prevalence in younger males. Rarely it affects both eyes. We report a middle aged female who presented with uniocular swelling and was later on diagnosed as pansinusitis with bilateral orbital cellulitis. Aggressive therapy with antibiotics and surgical drainage saved the patient.

Inflammation of ocular tissues behind or posterior to the orbital septum is called orbital cellulitis. Orbital cellulitis differs from preseptal cellulitis by causing inflammation of the tissues proximal to the orbital septum. The clinical features of orbital cellulitis include, proptosis, generalized malaise, conjunctival chemosis, ocular pain, blurred vision, headaches, fever, lid swelling and ophthalmoplegia. ${ }^{[1]}$ The inflammation can cross to the other orbit through cavernous sinus. The condition can cause loss of ocular function and death. Therefore, it should be considered an emergency. Approximately $11 \%$ of cases of orbital cellulitis result in visual loss.[1,2]

\section{PRESENTATION OF CASE}

A 55-year-middle aged non-diabetic female presented with orbital swelling. She noticed a pimple around her nose 4 days prior to the onset of swelling in her right eye. She scratched the pimple with her fingers and subsequently developed swelling in her right eye (figure -1).

On examination: She was febrile, Heart rate- 100 beats/minute, BP-100/60 mm of $\mathrm{Hg}$, respiratory rate was -20 cycles/min. Local examination: there was oedema of upper and lower eyelids on the right side and mild swelling of the left eyelid. Pus discharge was present. Eye movements were preserved and were not painful. There was presence of maxillary and ethmoid sinus tenderness. Other systemic examination were normal.

On laboratory investigations: Haemoglobin was $10 \mathrm{gm} \%$, TLC was 30,300/dl with polymorphs $80 \%$ and lymphocytes $16 \%$, Platelets was 1.4 Lacs/dl. Liver function was normal. Prothrombin time and INR was within normal range. Kidney function test showed creatinine $2.4 \mathrm{mg} / \mathrm{dl}$ and Blood urea nitrogen $100 \mathrm{mg} / \mathrm{dl}$. HIV and Hepatitis $\mathrm{B}$ and $\mathrm{C}$ screening was negative. Fasting and post prandial blood sugar were within normal limits. Urine examination was normal. Blood culture after 48 hours showed no growth. Ultrasound of abdomen and pelvis was normal.
Corresponding Author:

Sourya Acharya,

Professor,

Department of Medicine, Datta Meghe Institute of Medical Sciences (Deemed to be University) Sawangi, Meghe, Wardha-442001, Maharashtra, India. E-mail: souryaacharya74@gmail.com

\section{DOI: $10.14260 / j e m d s / 2020 / 319$}

Financial or Other Competing Interests: None.

How to Cite This Article:

Nayak A, Acharya S, Ghule A. Bilateral orbital cellulitis with pan sinusitis in a female without cavernous sinus thrombosis- a rare presentation. J. Evolution Med. Dent. Sci. 2020;9(17): 14641466, DOI: $10.14260 /$ jemds/2020/319

Submission 22-11-2019,

Peer Review 11-04-2020,

Acceptance 18-04-2020,

Published 27-04-2020.

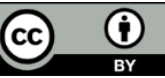




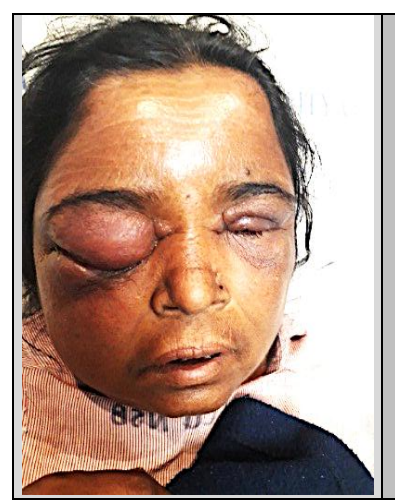

Figure 1. Gross Oedema of the Right Eye Lid and Mild Oedema of the Left Eye Lid

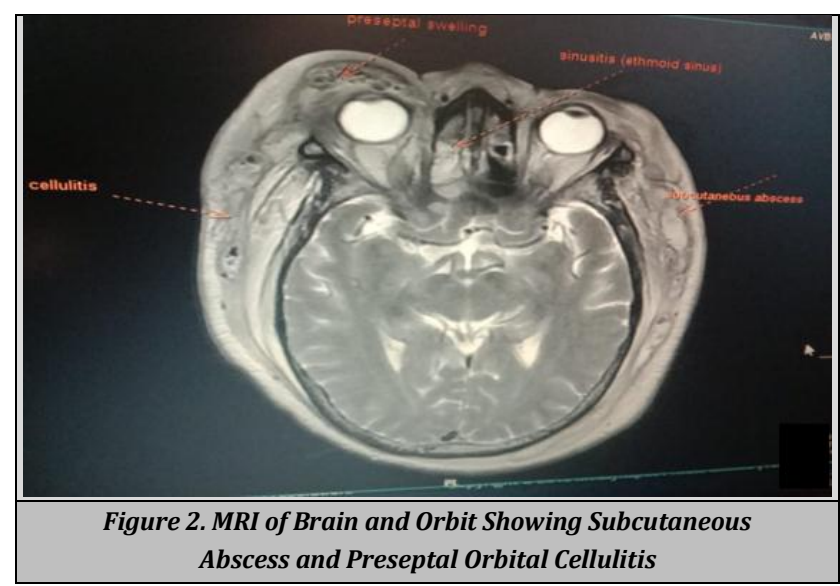

An initial diagnosis of right orbital cellulitis was constructed. She was treated with Inj. Piperacillin and Tazobactam 4.5 grams IV 6 hourly PLUS Inj. Clindamycin 900 mg IV 8 hourly for 2 weeks and intra venous fluids.

MRI orbit and brain showed bilateral Preseptal orbital cellulitis with subcutaneous abscess extending into the subgaleal space along with pan sinusitis and bilateral mastoiditis. There was no evidence of cerebral sinus venous thrombosis. A contrast study could not be obtained due to deranged creatinine levels. Culture and sensitivity of pus showed growth of staphylococcus aureus.

ENT and ophthalmology opinions were taken and urgent incision and drainage of the abscess was advised. Prompt surgical intervention and aggressive antibiotic treatment played a key role in resolution of cellulitis.

\section{DISCUSSION}

Orbital cellulitis is a life-endangering infection of the post septal tissues of the orbit commonly occurring in younger


result in loss of vision. [3]

It is believed to occur as a result of spread of infection from the bloodstream, adjoining sinuses, and facial skin.[2] Most cases involve only one eye, the second eye is only affected in presence of cavernous sinus involvement. The Postulated mechanism for bilateral orbital cellulitis suggests spread of infection through the ophthalmic veins to the cavernous sinus and centrifugally to the opposite orbit through the dura.[4],[5] The various classifications of orbital cellulitis have been influenced by involvement of cavernous sinus thrombosis. But interestingly our patient did not have cavernous sinus thrombosis/infection.[6,7] The recent classifications, are adaptations of the classifications used by Chandler et al. ${ }^{[6]}$ and Moloney et al. ${ }^{[8]}$ Incorporation of radiologic findings into the Moloney classification has been proposed in recent subject related proposals.[9]

Clinically, orbital cellulitis can present with pain, double vision or vision loss, proptosis, globe displacement, chemosis and ophthalmoplegia. Headache is a common accompaniment. In children, the incidence of fever is equal to that in preseptal cellulitis (62\%).[10] while it can be absent in adults $66 \%$ of the cases.[11]

Management of orbital cellulitis warrants inpatient hospitalization with urgent medical intervention. ${ }^{[2],[3] \mathrm{A}}$ workup including a computerized tomography scan (CT scan), full blood count analysis and blood culture should be obtained prior to starting the empirical therapy.[2],[3] Discharge from the nose and eyes are collected for microscopy, culture, and sensitivity.[3] Magnetic resonance imaging (MRI) is performed to confirm a diagnosis of orbital abscess and determine involvement of the cavernous sinus. ${ }^{[3]}$

Treatment includes aggressive administration of broadspectrum antibiotics and drainage of sub periosteal or orbital abscess whenever necessary.[2]

\section{CONCLUSIONS}

Orbital cellulitis is a catastrophic medical emergency. Ethmoid sinusitis may predispose to its development. Bilateral orbital cellulitis usually is associated with cerebral venous sinus thrombosis. Prompt neuroimaging, intravenous antibiotics and urgent surgical intervention may lead to its cure.

\section{REFERENCES}

[1] Wulc AE. Orbital infections. Chapter - 34. In: Linberg J, edr. Duane's Ophthalmology. CD-ROM ed, clinical vol 2. Philadelphia: Lippincot Williams and Wilkinson 2002.

[2] Kanski JJ. Orbit in clinical ophthalmology. $5^{\text {th }}$ edn. Edinburgh, New York: Butterworth Heinemann 2003: p. 568-9.

[3] Harrington JN. Orbital Cellulitis. http://emedicine.medscape.com/article/1217858.

[4] Eustis HS, Mafee MF, Walton C, et al. MR imaging and CT of orbital infections and complications in acute rhinosinusitis. Radiol Clin North Am 1998;36(6):1165-83.

[5] Singh SK, James E, Sabarigirish K, et al. Bilateral orbital complications of paediatric rhinosinusitis. Med J Armed Forces India 2014;70(1):68-72.

[6] Chandler JR, Langenbrunner DJ, Stevens ER. The pathogenesis of orbital complications in acute sinusitis. Laryngoscope 1970;80(9):1414-28.

[7] Smith AF, Spencer JF. Orbital complications resulting from lesions of the sinuses. Ann Otol Rhinol Laryngol 1948;57(1):5-27.

[8] Moloney JR, Badham NJ, McRae A. The acute orbit. Preseptal (periorbital) cellulitis, subperiosteal abscess 
and orbital cellulitis due to sinusitis. J Laryngol Otol Suppl 1987;12:1-18.

[9] Mortimore S, Wormald PJ. The Groote Schuur hospital classification of the orbital complications of sinusitis. J Laryngol Otol 1997;111(8):719-23.
[10] Schramm VL Jr, Curtin HD, Kennerdell JS. Evaluation of orbital cellulitis and results of treatment. Laryngoscope 1982;92(7 Pt 1):732-8.

[11] Bergin DJ, Wright JE. Orbital cellulitis. Br J Ophthalmol 1986;70(3):174-8. 